\title{
Reactor neutrino applications and coherent elastic neutrino nucleus scattering
}

\author{
Maitland Bowen ${ }^{1,2, *}$ and Patrick Huber ${ }^{1, \dagger}$ \\ ${ }^{1}$ Center for Neutrino Physics, Virginia Tech, Blacksburg, VA 24061 \\ ${ }^{2}$ University of Michigan, Ann Arbor, MI, 48109
}

(Dated: May 25, 2020)

\begin{abstract}
Potential applications of neutrino detection to nuclear security have been discussed since the 1970s. Recent years have seen great progress in detector technologies based on inverse beta decay, with the demonstration of ton-scale surface-level detectors capable of high quality neutrino spectrum measurements. At the same time coherent elastic neutrino nucleus scattering has been experimentally confirmed in 2017 with neutrinos from stopped pion decay and there is a number of experiments aimed at seeing this reaction with reactor neutrinos. The large cross section and threshold-less nature of this reaction make it plausible to consider it for applications to nuclear security and here, we present a first direct comparison of the two reaction modes.
\end{abstract}

\section{INTRODUCTION}

Neutrinos were discovered by Cowan and Reines in 1956 [1] using neutrino 1 from a nuclear reactor and inverse beta decay (IBD). Nuclear reactors are very bright neutrino sources with $10^{19}$ neutrinos released per second for $100 \mathrm{MW}$ of thermal reactor power. The neutrinos originate in the beta decays of neutron-rich fission fragments and not in the fission process itself. As a consequence, the neutrino spectrum and rate are sensitive to the fissioning isotope via the different fission fragment yields. In the 1970s, Mikaelyan [2] realized that this sensitivity could be exploited to learn about the reactor state by neutrino observations, giving rise to the field of applied neutrino physics, for a recent review see [3]. One of the major challenges arises from the fact that detectors for this application have to be able to work at the Earth's surface and be able to suppress the resulting backgrounds sufficiently to extract a high-fidelity signal. Detectors with the requisite characteristics have been demonstrated only very recently in 2018 [4, 5], more than 60 years after the initial detection of reactor neutrinos.

Coherent elastic neutrino nucleus scattering (CEvNS) was postulated in $1974[\underline{6}$ and experimentally confirmed in 2017 by the COHERENT experiment [7] using neutrinos with 10s of $\mathrm{MeV}$ energy. The CEvNS reaction is interesting for applications because the cross section per unit detector mass can be two orders of magnitude larger than for IBD, potentially allowing for detectors in the kilogram range. This reaction is also thresholdless, potentially providing access to safeguards-relevant signatures of plutonium breeding [8]. The detection of reactor neutrinos, which have a mean energy of $4 \mathrm{MeV}$, via CEvNS has not yet been demonstrated, but there are several experiments aimed at this goal [9 14].

\footnotetext{
${ }^{1}$ In this letter we deal exclusively with electron antineutrinos and for brevity will refer to them simply as neutrinos.
}

Physicists in the Soviet Union in 1978 first proposed the use of neutrinos for remote monitoring of nuclear reactors [2]. The typical mixture of isotopes undergoing fission in a reactor comprises ${ }^{235} \mathrm{U},{ }^{238} \mathrm{U},{ }^{239} \mathrm{Pu}$, and ${ }^{241} \mathrm{Pu}$. The plutonium content is a result of breeding reactions, which happen in all reactors fueled with natural or lowenriched uranium and proceeds via neutron captures and beta decays. Each of these isotopes also has well-defined and unique neutrino emissions, and both the energy spectra and the number of neutrinos are different [15, 16]. Plutonium-239 has the lowest average neutrino energy of the four isotopes within the reactor and this characteristic allows for the determination of the plutonium content of the reactor. The number of neutrinos emitted for each isotope is also different and the resulting differences in IBD event rates are shown in Tab. I By observing the number of neutrinos emitted by reactors and the spectra of those neutrinos, the composition of the reactor fuel and the power level of the reactor can be determined. As a result, one can deduce whether the reactor could potentially be producing weapons-grade material, even without access to records of the reactor history [17].

Analyses with individual reactors have demonstrated that this method of safeguards would have provided timely information as to the plutonium production in the Democratic People's Republic of Korea during the North Korean nuclear crisis of 1994, even given the limited access inspectors were given to the reactor [17]. Studies have also applied this method to IR-40, the Iranian heavy water reactor at Arak, and demonstrated that a neutrino detector can meet or exceed the verification goals set by the International Atomic Energy Agency (IAEA) [18].

Potential applications are not limited to reactor power monitoring and plutonium production monitoring but could include detection of nuclear waste streams from reprocessing and spent nuclear fuel [17, 19], as well as long-distance detection, see also Ref. [3]. For the two applications considered here, IBD detectors in mass range of tons are sufficient and detectors with requisite capabilities have been experimentally demonstrated. At the same time, the neutrino emission spectra for both re- 
actors and nuclear wastes have most of their emission below the IBD threshold. In particular, the neutron capture pathway from uranium to plutonium at a reactor includes beta decays which produce neutrinos of less than 1.2 MeV. By number, the flux of these "breeding" neutrinos exceeds the flux of fission fragment neutrinos in that energy range significantly. Therefore, some applications would benefit significantly if a threshold-less reaction could be exploited [8].

\section{RATES \& SPECTRA}

In IBD an electron antineutrino scatters off a proton and produces a neutron and positron

$$
\bar{\nu}_{e}+p \rightarrow n+e^{+}
$$

Since the neutron is heavier than the proton this reaction has a threshold energy, which for the proton at rest is given by

$$
E_{\nu}^{t h r}=\frac{\left(M_{N}+m_{e^{+}}\right)^{2}-M_{p}^{2}}{2 M_{p}}=1.806 \mathrm{MeV}
$$

Since $m_{e}, m_{\nu} \ll m_{n}, m_{p}$, the energy of the incoming neutrino and positron energy have a one-to-one relation $E_{e^{+}}=E_{\nu}-E_{\nu}^{t h r}$. The total cross section at zeroth order can be expressed as

$$
\sigma=\frac{2 \pi}{m_{e}^{5} f^{R} \tau_{n}} E_{e} p_{e}
$$

where $\tau_{n}$ is the measured neutron lifetime and $f^{R}=1.7152$ is the phase space factor [20]. The cross section for IBD for reactor neutrinos is approximately $6 \times 10^{-43} \mathrm{~cm}^{2}$ per fission. To compute the neutrino event rates and energy spectra from IBD, we use the cross section from Ref. [20] and antineutrino fluxes for ${ }^{235} \mathrm{U},{ }^{238} \mathrm{U},{ }^{239} \mathrm{Pu}$, and ${ }^{241} \mathrm{Pu}$ from a simple summation calculation based on the data in Ref. [16]. The use of this summation calculation allows us to extend the neutrino spectrum to energies below the IBD threshold, which is needed for the CEvNS calculations. Note, that this summation fluxes, as is usual, deviate by about $5 \%$ from the Huber-Mueller fluxes [15, 16]; however, the relative properties of the four fissile isotopes are a robust feature [17].

We compute the neutrino yield for each isotope through numerical integration. As a benchmark we chose a $100 \mathrm{MW}_{\text {th }}$ reactor, $1 \mathrm{~kg} \mathrm{CH}_{2}$ detector, $10 \mathrm{~m}$ standoff from the reactor core and a data taking period of one year. $\mathrm{CH}_{2}$ is a proxy for an actual organic scintillator but approximates the proton fraction of most scintillators. Note, that $100 \mathrm{MW}_{\text {th }}$ is typical of plutonium production reactors and thus is a relevant bench mark. The results are shown in Tab. [1] the number of neutrinos detected with IBD from each isotope in a reactor differs

$$
\begin{array}{c|rrrr}
\text { Isotope } & { }^{239} \mathrm{Pu} & { }^{241} \mathrm{Pu} & { }^{235} \mathrm{U} & { }^{238} \mathrm{U} \\
\hline \text { Events } & 288 & 398 & 418 & 636
\end{array}
$$

TABLE I. IBD event number per $\mathrm{kg}$ of $\mathrm{CH}_{2}$ per year at a $100 \mathrm{MW}_{\mathrm{th}}$ reactor and at a distance of $10 \mathrm{~m}$.

significantly. At approximately 300 events with our given parameters, ${ }^{239} \mathrm{Pu}$ produces the fewest neutrinos, at only about two-thirds the rate of ${ }^{238} \mathrm{U} .{ }^{239} \mathrm{Pu}$ also has the lowest mean energy; ${ }^{238} \mathrm{U}$ has the highest mean energy. With observations of both the event number and the spectra of the neutrinos emitted from a nuclear reactor, one can deduce both the composition of the mixture within the reactor and the power level of the reactor itself.

Coherent elastic neutrino-nucleus scattering (CEvNS) was postulated soon after neutral currents were discovered; it occurs between a neutrino of any flavor and a target nucleus [6]

$$
\bar{\nu}+X \rightarrow \bar{\nu}+X
$$

where the signature is the recoil of the target nucleus $X$. The cross section is approximately given by

$$
\frac{d \sigma}{d T}\left(E_{\nu}\right)=\frac{G_{F}^{2}}{4 \pi} N^{2} M\left(1-\frac{M T}{2 E_{\nu}^{2}}\right)
$$

where we have neglected any nuclear form factors; for reactor neutrino energies this is an excellent approximation. $N$ is the neutron number, $M$ is the nuclear mass and $T$ the nuclear recoil energy. CEvNS holds promise for low-energy neutrinos detection due to the $N^{2}$ dependence. Despite its high cross section, CEvNS evaded detection for decades because of the difficulty in detecting very low nuclear recoil energies. In 2017, the COHERENT collaboration used a $14.6 \mathrm{~kg}$ CsI[Na] scintillator detector to observe CEvNS for the first time from neutrinos at the Spallation Neutron Source at Oak Ridge National Laboratory [7]. The relation between the observable recoil energy $T$ and neutrino energy $E_{\nu}$ is not one-to-one and is more similar to the case of Compton-scattering, therefore the neutrino energy spectrum information is less direct than in the IBD case.

For CEvNS we use the cross section in Eq. 4 and the same reactor parameters and standoff as for IBD. The relevant observable is the nuclear recoil energy $T$ and for a given neutrino energy there is a kinematic limit to what the maximum recoil energy can be

$$
T_{\max }=\frac{E_{\nu}}{1+\frac{M_{N}}{2 E_{\nu}}}
$$

Unlike IBD, CEvNS can occur on any target and thus there is a wide range of potential detector materials. We show results for a selection of commonly used target materials. The cross section peaks at low values of $T$ and therefore, the total event rate sensitively depends on the 
low-energy detection threshold for nuclear recoils, $T_{\min }$. One common way to quote CEvNS cross sections is per target nucleus but for practical application the actual target mass is more critical. Table III shows the neutrino event number above a given nuclear recoil energy threshold for various target materials that could serve in detectors for CEvNS. Note, that we sum the contributions of each stable isotope of each element weighted by its natural abundance.

\begin{tabular}{c|r|r|r|r|r|r|r} 
Threshold $[\mathrm{eV}]$ & $\mathrm{C}$ & $\mathrm{Ne}$ & $\mathrm{Si}$ & $\mathrm{Ar}$ & $\mathrm{Ge}$ & $\mathrm{Xe}$ & $\mathrm{W}$ \\
\hline 0 & 1255 & 2147 & 2958 & 5048 & 9526 & 19033 & 27406 \\
10 & 1223 & 2058 & 2794 & 4669 & 8343 & 15270 & 20462 \\
100 & 1023 & 1565 & 1954 & 2908 & 3913 & 4623 & 4350 \\
1000 & 335 & 296 & 227 & 169 & 35 & 1 & 0
\end{tabular}

TABLE II. CEvNS event number per kg per year for fission of ${ }^{235} \mathrm{U}$ at a $100 \mathrm{MW}_{\text {th }}$ reactor and at a distance of $10 \mathrm{~m}$ as a function of isotope and recoil energy threshold.

Table III shows the recoil energies at which a CEvNS detector with the given isotope as a target will detect the same number of neutrinos as an IBD detector of the same mass. This is again using the parameters outlined previously with respect to reactor power, observation time, distance, and target mass for ${ }^{235} \mathrm{U}$. For example, a germanium detector will detect fewer neutrinos than a currently operating IBD detector if the CEvNS detector is unable to observe recoil energies below the required $496 \mathrm{eV}$. Table III also demonstrates the linearity of the threshold energy versus the mass number of the target. Note that we are specifically quoting the nuclear recoil energy, the electron-equivalent measured energy typically is much lower due to quenching.

This allows us to make a first observation: almost every potential target isotope we considered will detect fewer neutrinos than a currently operating IBD detector if the CEvNS detector is unable to observe recoil energies below $1 \mathrm{keV}$. To put this in context, the original COHERENT observation was achieved with a recoil threshold of approximately $5 \mathrm{keV}$. It is important to point out that what matters here is the nuclear recoil threshold where a reactor CEvNS event can be identified at a reasonable level of background and with good efficiency. Consider for example the recent CONNIE result [21], where the detector has a threshold of $64 \mathrm{eV}$, but only at around $200 \mathrm{eV}$ the median efficiency is reached and once quenching is taken into account, this corresponds to $\sim 1 \mathrm{keV}$ recoil threshold.

$$
\begin{array}{c|rrrrrrr}
\text { Isotope } & \mathrm{C} & \mathrm{Ne} & \mathrm{Si} & \mathrm{Ar} & \mathrm{Ge} & \mathrm{Xe} & \mathrm{W} \\
\hline T_{\min }[\mathrm{eV}] & 791 & 782 & 707 & 677 & 496 & 352 & 281
\end{array}
$$

TABLE III. The recoil energy threshold at which IBD and the CEvNS detection result in the same neutrino event number.

Unlike IBD, CEvNS does not have a one-to-one corre-

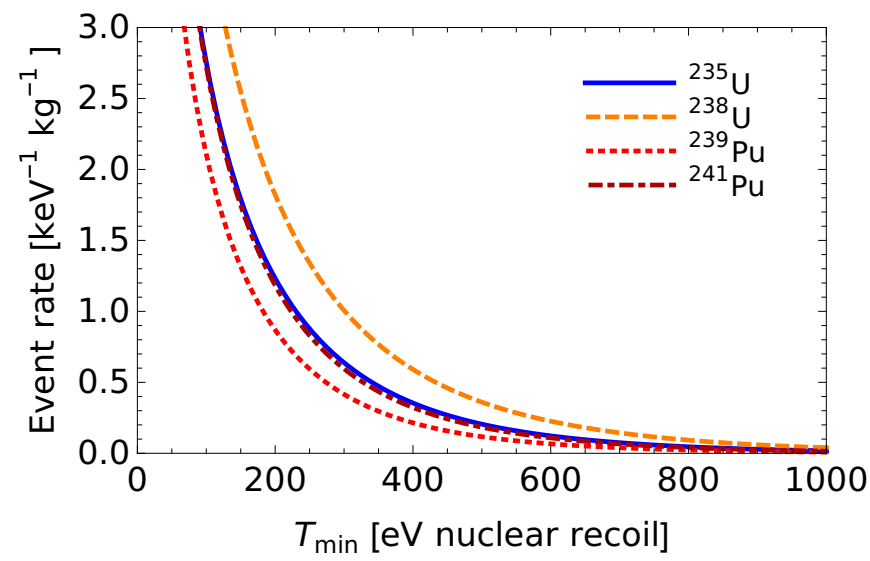

FIG. 1. Shown is the nuclear recoil spectrum on germanium in arbitrary units for neutrinos stemming from fission of ${ }^{235} \mathrm{U}$, ${ }^{239} \mathrm{Pu},{ }^{238} \mathrm{U}$, and ${ }^{241} \mathrm{Pu}$, respectively.

spondence between the energy deposited in the detector and the true neutrino energy. For applications, the potential to track plutonium production is entirely due to the different energy spectra of the different fissile isotopes. In Fig. 1 we show the recoil energy spectrum in germanium for the usual fissile isotopes. The spectral differences persist also in the recoil spectrum and are most prominent in the $100-200 \mathrm{eV}$ region.

\section{APPLICATION RELEVANCE}

Research in IBD applications has come a long way since the original proposal in 1978 and we take the results of Ref. 22], which in turn is based on the performance of the PROSPECT detector [4], as our benchmark in reference to two scenarios relevant to nuclear security:

- Reactor power: How long does it take to observe a transition from reactor on to off or vice versa? With IBD, this can be achieved with 1-2 tons of active detector mass over a period of hours to days.

- Plutonium production: How long does it take to distinguish a new, plutonium-free, reactor core from an old, plutonium-rich, core? With IBD, this can be achieved with active detector masses of 1020 tons in a period of weeks to months for most reactor types.

It is of note, that those previous result do include real backgrounds as measured by PROSPECT and that realistic deployment scenarios have been considered.

CEvNS at reactors has not been yet observed and it is clear that apart from achieving a low nuclear recoil threshold, mitigation of backgrounds will be the main challenge, see e.g. Ref. 11]. Apart from the signal to background $(\mathrm{S}: \mathrm{B})$ ratio also the shape of the background matters, especially for $S: B<1$. In Fig. 2 we show 


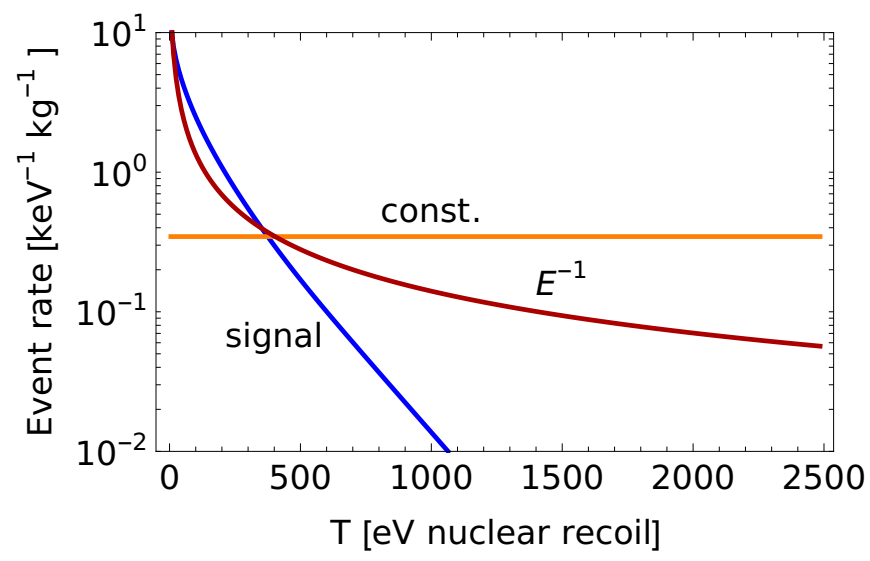

FIG. 2. Shown are potential background models for the CEvNS process.

the signal shape in comparison to a number of plausible background shapes. In reality the background will be a mix of shapes since several independent processes will contribute. Clearly, a $E^{-1}$ background is the most pernicious, yet seems to be similar to what many experiments see at very low recoil thresholds, see Ref. 23] for a recent compilation of low-energy backgrounds.

Having fixed the shape of our background model the only remaining free parameters are S:B and the recoil energy threshold. We use the same analysis framework as Ref. [22] and can now evaluate what the ratio of the measurement precision for either reactor power or plutonium content is for a fixed mass of IBD and CEvNS detectors. The employed likelihood function is quadratic in the parameters of interest and hence all precision scales as the inverse square of detector mass. Therefore, the inverse square of the ratio of precision for a given measurement expresses how many times smaller or larger a CEvNS detector would need to be to match the capabilities of an IBD detector; we call this quantity the mass advantage. If we neglect backgrounds, we find that the mass advantage becomes unity for about one half the recoil energy threshold values listed in Tab. III]

This result applies for both power and plutonium content measurements. This arises from less pronounced spectrum differences between fissile isotopes in CEvNS. The reason this also affects the power measurements is the degeneracy between reactor power and plutonium content [17]. In the ideal background-free case the mass advantage for xenon can reach 40 for plutonium-content determination and up to 80 for power monitoring, albeit at a recoil threshold of $5 \mathrm{eV}$. At the other end of atomic masses, the mass advantage for silicon is $3-6$ at best.

Taking into account background, we obtain the results shown in Fig 3, which are computed for sodium, germanium, and xenon for a plutonium content measurement. For $\mathrm{S}: \mathrm{B}=1$, which is essentially what has been demonstrated for IBD, we find that a mass advantage of unity

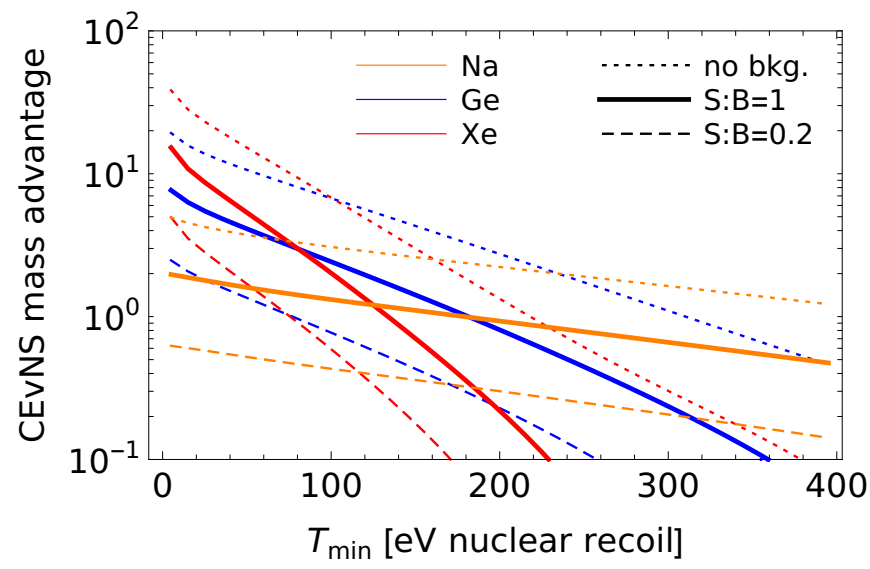

FIG. 3. Shown is the ratio of detector mass between CEvNS and IBD to achieve the same precision as the corresponding IBD measurement of the reactor plutonium content, including a $1 / E$ background component with the indicated $\mathrm{S}: \mathrm{B}$ ratio.

is reached for a recoil threshold of about $140-300 \mathrm{eV}$. The mass advantage climbs to a value of $8-12$ for a $5 \mathrm{eV}$ recoil threshold. Should the background be 5 times or more larger than the signal, it becomes very difficult to obtain any mass advantage.

\section{SUMMARY}

We have presented a first comparison of the event rates, spectra and resulting reactor monitoring capabilities of inverse beta decay and the recently confirmed CEvNS process. We find that a nuanced picture arises when we consider both the energy threshold for the detection of nuclear recoil and the actual detector mass, instead of the cross section per target nucleus. To achieve detected event rates per unit detector mass on par with IBD, recoil thresholds of $300-800 \mathrm{eV}$ are necessary. The information contained in the neutrino energy spectrum regarding reactor plutonium content does persist in CEvNS, albeit at a lower level due to the unknown energy carried away by the outgoing neutrino. In a direct comparison of the resulting ability to measure reactor power and the plutonium content, we find that CEvNS detectors must achieve recoil energy thresholds as low as $200 \mathrm{eV}$ to be similar to IBD detectors. Taking into account backgrounds, we find that CEvNS detectors at best offer a mass advantage of one order of magnitude assuming that $\mathrm{eV}$-scale recoil thresholds are feasible and a signal to background ratio of about 1 can be achieved. In our comparison we did not consider the deployed weight of the entire detector system or the ability to run remotely with no user intervention for extended periods of time, which, for instance, might present a challenge for cryogenic detectors. Based on those results, it appears to us that, for the foreseeable future, the best use case for CEvNS is not 
to replace IBD detectors but to complement them, for instance with the direct detection of neutrinos produced in plutonium breeding [8].

\section{ACKNOWLEDGMENTS}

We thank P. Barbeau and K. Schollberg for useful discussions. The work of MB was supported by the National Science Foundation REU grant number 1757087. The work of PH was supported by the U.S. Department of Energy Office of Science under award number de-sc00018327 and the National Nuclear Security Administration Office of Defense Nuclear Nonproliferation R\&D through the consortium for Monitoring, Technology and Verification under award number de-na0003920.

* mebowen@umich.edu

† pahuber@vt.edu

[1] C. L. Cowan, F. Reines, F. B. Harrison, H. W. Kruse, and A. D. McGuire, "Detection of the free neutrino: A Confirmation," Science 124, 103-104 (1956).

[2] A. A. Borovoi and L. A. Mikaelyan, "Possibilities of the practical use of neutrinos," Soviet Atomic Energy 44, 589 (1978).

[3] Adam Bernstein, Nathaniel Bowden, Bethany L. Goldblum, Patrick Huber, Igor Jovanovic, and John Mattingly, "Colloquium: Neutrino detectors as tools for nuclear security," Rev. Mod. Phys. 92, 011003 (2020), arXiv:1908.07113 [physics.soc-ph]

[4] J. Ashenfelter et al. (PROSPECT), "First search for short-baseline neutrino oscillations at HFIR with PROSPECT," Phys. Rev. Lett. 121, 251802 (2018), arXiv:1806.02784 [hep-ex],

[5] Alireza Haghighat, Patrick Huber, Shengchao Li, Jonathan M. Link, Camillo Mariani, Jaewon Park, and Tulasi Subedi, "Observation of Reactor Antineutrinos with a Rapidly-Deployable Surface-Level Detector," Phys. Rev. Applied 13, 034028 (2020), arXiv:1812.02163 [physics.ins-det].

[6] Daniel Z. Freedman, "Coherent Neutrino Nucleus Scattering as a Probe of the Weak Neutral Current," Phys. Rev. D9, 1389-1392 (1974).

[7] D. Akimov et al. (COHERENT), "Observation of Coherent Elastic Neutrino-Nucleus Scattering," Science 357, 1123-1126 (2017), arXiv:1708.01294 [nucl-ex].

[8] Bernadette K. Cogswell and Patrick Huber, "Detection of breeding blankets using antineutrinos," Science \& Global Security 24, 114-130 (2016). https://doi.org/10.1080/08929882.2016.1184531.

[9] G. Agnolet et al. (MINER), "Background Studies for the MINER Coherent Neutrino Scattering Reactor Ex- periment," Nucl. Instrum. Meth. A853, 53-60 (2017), arXiv:1609.02066 [physics.ins-det].

[10] J. Billard et al., "Coherent Neutrino Scattering with Low Temperature Bolometers at Chooz Reactor Complex," J. Phys. G44, 105101 (2017), arXiv:1612.09035 [physics.ins-det].

[11] J. Hakenmüller et al., "Neutron-induced background in the CONUS experiment," $\quad$ Eur. Phys. J. C 79, 699 (2019), arXiv:1903.09269 [physics.ins-det].

[12] D. Yu Akimov et al. (RED-100), "First groundlevel laboratory test of the two-phase xenon emission detector RED-100," JINST 15, P02020 (2020) arXiv:1910.06190 [physics.ins-det].

[13] Alexis Aguilar-Arevalo et al. (CONNIE), "Exploring low-energy neutrino physics with the Coherent Neutrino Nucleus Interaction Experiment," $\quad$ Phys. Rev. D100, 092005 (2019) arXiv:1906.02200 [physics.ins-det].

[14] G. Angloher et al. (NUCLEUS), "Exploring $\mathrm{CE} \nu \mathrm{NS}$ with NUCLEUS at the Chooz nuclear power plant," Eur. Phys. J. C79, 1018 (2019), arXiv:1905.10258 [physics.ins-det].

[15] Th. A. Mueller et al., "Improved predictions of reactor antineutrino spectra," Phys. Rev. C 83, 054615 (2011), arXiv:1101.2663 [hep-ex]

[16] Patrick Huber, "On the determination of anti-neutrino spectra from nuclear reactors," $\quad$ Phys.Rev. C84, 024617 (2011) arXiv:1106.0687 [hep-ph],

[17] Eric Christensen, Patrick Huber, and Patrick Jaffke, "Antineutrino reactor safeguards - a case study," Science and Global Security 23, 20-47 (2015), arXiv:1312.1959 [physics.ins-det].

[18] Eric Christensen, Patrick Huber, Patrick Jaffke, and Thomas E. Shea, "Antineutrino Monitoring for Heavy Water Reactors," Phys. Rev. Lett. 113, 042503 (2014), arXiv:1403.7065 [physics.ins-det]

[19] Vedran Brdar, Patrick Huber, and Joachim Kopp, "Antineutrino monitoring of spent nuclear fuel," Phys. Rev. Applied 8, 054050 (2017), [Phys. Rev. Applied.8,054050(2017)], arXiv:1606.06309 [hep-ph]

[20] P. Vogel and John F. Beacom, "Angular distribution of neutron inverse beta decay," $\quad$ Phys. Rev. D60, 053003 (1999), arXiv:hep-ph/9903554 [hep-ph].

[21] Alexis Aguilar-Arevalo et al. (CONNIE), "Search for light mediators in the low-energy data of the CONNIE reactor neutrino experiment," JHEP 04, 054 (2020), arXiv:1910.04951 [hep-ex].

[22] Rachel Carr et al., "Neutrino-based tools for nuclear verification and diplomacy in North Korea," Science \& Global Security 27, 15-28 (2018) arXiv:1811.04737 [physics.soc-ph]

[23] Noah Kurinsky, Daniel Baxter, Yonatan Kahn, and Gordan Krnjaic, "A Dark Matter Interpretation of Excesses in Multiple Direct Detection Experiments," (2020), arXiv:2002.06937 [hep-ph] 